\title{
Replaced Loss of Upper Eyelid by Frontal Skin Graft
}

Dieu $\mathrm{D}^{*}$

Dean of Optical Ophthalmology, Faculty-Nguyen Tat Thanh University, VietNam.

\section{Introduction}

Loss of an upper eyelid can arise through trauma in local/remote area $[1,2]$. This condition in which the eye cannot be closed is severe affected patient's vision with unavailable treatment [3-6]. The patient should be transferred to tertiary health care level for treatment. We introduce the simple surgical techniques that can be performed at the second health care level under local anesthesia without the need for expensive technical equipment. This is an auto frontal skin grafting-single staged procedure on the course of hospital administration for correction of upper lid which was cut out off due to trauma.

\section{Patient \& Method}

\section{Surgical Techniques}

To reconstruct new eyelid by graft of frontal skin flap was done.

1. An incision was made to create a crescent-shaped forehead skin flap $(70 \times 20 \mathrm{~mm})$ with a few lines of eye- brow (Figure. 2).

2. The pedicle was at the lateral level of the eyebrow. This skin flap was used to replace the upper eyelid. The few lines of eyebrow replaced the lost eyelashes.

3. The forehead wound beneath the flap was closed with running or interrupted sutures (Figure. 3).

4. To reconstruct a new upper eyelid, the skin flap was rotated anticlockwise to the remaining left upper lid. Using interrupted sutures the head flap was sutured to the medial canthus, the upper edge of the flap to the residual upper lid. The lower edge of the flap with the few lines of eyebrow was turned down to become the new eyelid margin using running sutures side-by-side (Figure 3). The pedicle remained and was sufficient to support the skin graft.

One week post operation these sutures were cut off.

\section{Results}

\section{Case Report}

A 32-year-old male farmer fell while digging. He had suddenly known bleeding from left eye and severe headache, so he cried for helping. The neighbor took him to health station of village. A first aid dressing was applied at the local primary health care center and the patient was admitted to the provincial hospital (Figure 1).

Ocular examination revealed a visual acuity of $20 / 20$ in the right eye (RE) and 20/20 in the left eye (LE). Intraocular pressure was $12 \mathrm{mmHg}$ in both eyes. LE: The upper eyelid was almost completely severed together with all the eyelashes, three-quarters of the skin, two-thirds of the tarsus, and half of the conjunctiva of the upper lid from lid margin. Both eyeballs were normal. General examination: Nothing abnormal detected. Patient's history: well-being.

$\mathrm{BP}=120 / 70 \mathrm{mmHg} \cdot \mathrm{BG}=80 \mathrm{mg} \% ; \mathrm{BT}=2^{\prime} \mathrm{BC}=5^{\prime}$.

\section{Diagnosis}

$\mathrm{RE}=$ Normal. $\mathrm{LE}=$ Loss of upper lid due to trauma.

\section{Surgical Treatment}

$\mathrm{LE}=$ Surgery was done with local anesthesia by injection $10 \mathrm{ml}$ of lidocaine $2 \%$ into the three branches of the trigeminal nerve: frontal, nasal and lacrimal sites from the supraorbital to provide sensory innervation for the skin of the forehead and scalp. The skin flap from frontal skin $(70 \times 20 \mathrm{~mm})$ with a few lines of eyebrow was created. To reconstruct a new upper eyelid by using this flap rotated anticlockwise to the remaining left upper lid (Figure 2). This forehead wound beneath the flap was closed with interrupted sutures.

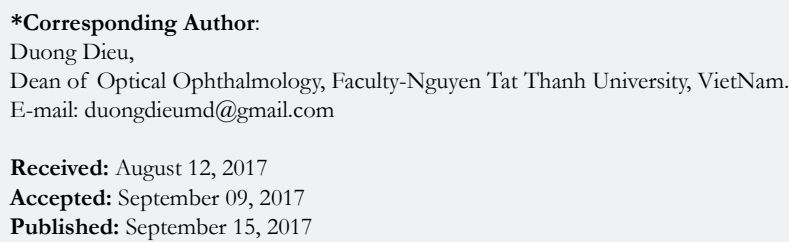


Using the head flap was sutured to the medial canthus, the upper edge of the flap to the residual upper lid by running sutures. The lower edge of the flap with the few lines of eyebrow was turned down to become the new eyelid margin, using running sutures side-by-side (Figure 3).

\section{Post-operative Recovery}

By the second or third week postoperatively, bruising had diminished and the color of the head skin flap had regained normal. Visual acuity for this patient 6 months later remained at $20 / 20$.

\section{Discussion}

Loss of an upper eyelid results in aesthetic and functional problems $[4,6]$.

A variety of inventive techniques are available to repair large to total upper eyelid defects $[3,5]$. These techniques include the CutlerBeard bridge flap from the lower eyelid, the inverted semicircular flap, multiple composite eyelid grafts, the lower eyelid switch flap, the inferiorly based tarsoconjunctival flap, the tarsoconjunctival horizontal advancement flap, the tarsoconjunctival rotational flap, and medial or temporal forehead flaps [6]. Although excellent results can be obtained with these procedures, several disadvantages exist. Many of these procedures are 2-staged [4-6].

In our case, because of full thickness loss of the total upper eyelid, Mustarde's method of repair (pedicle flap from lower lid to upper lid) was not appropriate. Thus, a flap from the forehead was used, one-stage-procedure.

When traumatic cutting of the upper eyelid occurs, the levator muscle retracts into the orbital fat although it can be retrieved. Secondary repair of post traumatic ptosis should be delayed for 6 to 9 months as the condition may be self- limiting [1, 3].

In this patient with full-thickness defect of the upper lid caused by trauma, the levator muscle was not reconstructed. A forehead skin flap to upper lid was performed to cover the eyeball as soon as possible. This flap derives its blood supply from the superficial temporal artery that travels along the lateral orbital rim and above the brow in that area. The pedicle was big enough to nourish the flap. In the author's experience, the width of the pedicle should be one-third the length of the flap.

The advantage of this one-stage procedure is that the eyeball is

Figure 1. Loss of Upper Lid Due to Trauma.

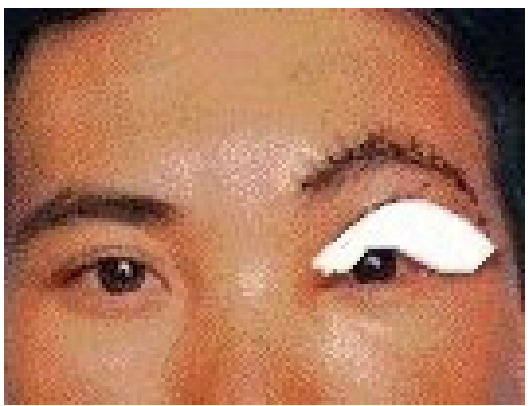

Figure 2. Auto Frontal Skin Graft (Red Line) to Replace Upper Lid Loss (Blue Arrow).

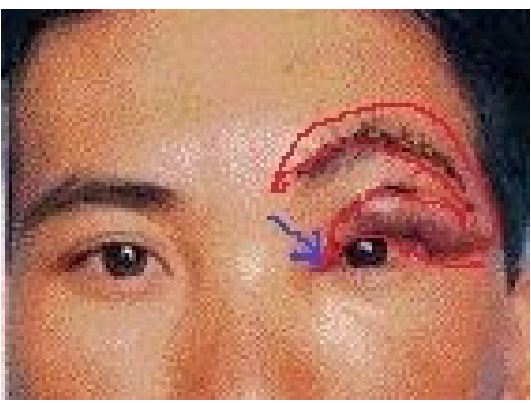

Figure 3. Frontal Skin Wound: Interrupted Sutures; New Upper Lid: Running Sutures.

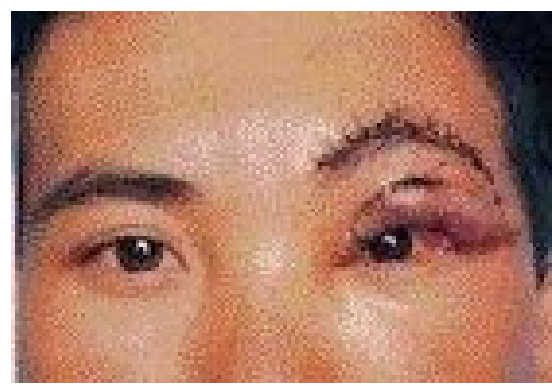


covered as soon as possible, thus preventing the possible sequelae of exposure such as ulceration of the conjunctiva or cornea, endophthalmitis, and rarely, enucleation [2].

In addition, in a tropical climate, ultraviolet light can cause lens and retinal damage. Ultimately, blindness may result. A disadvantage of this technique is that function of the levator muscle is not restored [4].

\section{Conclusion}

In our experience, auto skin grafting, single-staged procedureon the course of hospital administration for correction of upper lid lagophthalmos will then improve visual acuity as well as appearance.

\section{References}

[1]. Garber P, McDonald D, Machule BC. Injuries to the adnexa. Ibid. 1987;417-469.

[2]. Bohigian GM. Handbook of external diseases of the eye. J pediatric Opthamol strabismus. 1987 Nov 1;24(6):26-33.

[3]. Siddens JD, Nesi FA. Management of acquired ptosis. Smith's Ophthalmic and Reconstructive Surgery. Nesi FA, Levine MR, editors. St. Louis: Mosby; 1998.

[4]. McCulley TJ, Kersten RC, Kulwwin DR, Fueur WJ. Outcome and influencing factors of external levator palpebrae superiosis advancement for blepharoptosis. Ophthal Plast Reconstr . Surg. 2003 9;19(5):338-393.

[5]. Cutler NL, Beard CA. Method for partial and total upper lid reconstruction. Am J Ophthalmol. 1955 Jan;39(1):1- 7.

[6]. Mustardé JC. Major reconstruction of the eyelids: functional and aesthetic considerations. Clin Plast Surg. 1981 Apr: 8(2):227-231. 